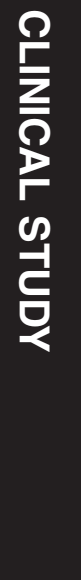

${ }^{1}$ Singapore Eye Research Institute, Singapore, Singapore

2Duke-NUS Graduate Medical School, Singapore, Singapore

${ }^{3}$ Bioinformatics Institute, A*STAR (Agency for Science, Technology and Research), Singapore, Singapore

${ }^{4}$ State Key Laboratory of Ophthalmology, Zhongshan Ophthalmic Center, Sun Yat-sen University, Guangzhou, China

${ }^{5}$ Singapore National Eye Centre, Singapore,

Singapore

${ }^{6}$ Yong Loo Lin School of Medicine, Department of Ophthalmology, National University of Singapore, Singapore, Singapore

Correspondence:

T Aung, Singapore National Eye Centre, 11 Third Hospital Avenue, Singapore 168751, Singapore Tel: +65 62277255 ; Fax: +65 62263395 .

E-mail: aung.tin@snec.com.sg

Received: 7 June 2016 Accepted in revised form: 14 October 2016 Published online: 9 December 2016

\title{
Anterior segment imaging-based subdivision of subjects with primary angle- closure glaucoma
}

\begin{abstract}
Purpose The purpose of this study was to identify whether it was possible to subdivide subjects with primary angle-closure glaucoma (PACG) based on anterior segment optical coherence tomography (ASOCT) imaging, and to determine the characteristics of such subgroups.

Methods We evaluated 210 subjects with PACG. All subjects underwent gonioscopy and ASOCT imaging. Customized software was used to measure ASOCT parameters. An agglomerative hierarchical clustering method was first used to determine the optimum number of parameters to be included in the determination of subgroups. Then, the best number of subgroups was determined using Akaike Information Criterion (AIC) and Gaussian Mixture Model (GMM) methods. Results The mean age of the subjects was 67.9 years, and $53.3 \%$ were female. Following the hierarchical clustering, four parameters (iris area, anterior chamber depth (ACD), anterior chamber width (ACW), and lens vault (LV)) were chosen to be representative of related parameters. The optimal number of subgroups using GMM analysis and AIC was 3. Subgroup $1(N=89 ; 42.4 \%)$ was characterized by a large iris area, subgroup 2 $(N=24 ; 11.4 \%)$ by a large $\mathrm{LV}$ and a shallow ACD, whereas subgroup $3(N=97 ; 46.2 \%)$ displayed only intermediate values across iris area, LV, and ACD.

Conclusions We identified three distinct subgroups of PACG subjects based on ASOCT imaging.
\end{abstract}

Eye (2017) 31, 572-577; doi:10.1038/eye.2016.267; published online 9 December 2016

\section{Introduction}

Primary angle-closure glaucoma (PACG), a major form of glaucoma in Asia, ${ }^{1}$ is a chronic
ME Nongpiur ${ }^{1,2}$, E Atalay ${ }^{1}, \mathrm{~T} \mathrm{Gong}^{3}, \mathrm{M} \mathrm{Loh}^{3}$, HK Lee ${ }^{3}, \mathrm{M} \mathrm{He}^{4}$, SA Perera ${ }^{1,5}$ and T Aung ${ }^{1,5,6}$ asymptomatic disease with higher blindness rates at presentation compared with primary open angle glaucoma. ${ }^{2,3}$ Laser peripheral iridotomy (LPI) is the first-line intervention for angle-closure (AC) disease, as it eliminates pupillary block. However, despite a significant increase in angle width post-LPI, one-fifth of eyes still display residual AC. ${ }^{4}$ Furthermore, the Zhongshan Angle-Closure Prevention Trial demonstrated that the increase in angle width after LPI remained stable for 6 months, and then decreased markedly by 18 months after LPI. ${ }^{5}$ These observations suggest that mechanisms other than pupil block contribute to AC and some change over time.

With the advances in imaging technology, it may be possible to obtain a better understanding of the mechanisms involved in PACG. It is becoming increasingly evident that $\mathrm{AC}$ is not caused by a single mechanism, rather it is influenced by forces acting at different anatomical levels that ultimately cause crowding of the angles. ${ }^{6-8}$ An ability to identify the predominant factor(s) in the development of AC may not only enhance our understanding of disease pathogenesis but may aid and improve our clinical management of patients with PACG.

We have previously shown that it was possible to subgroup primary angle-closure suspects (PACSs) based on anterior segment optical coherence tomography (ASOCT) imaging and clustering analysis into three distinct subgroups. One group was characterized by a thick peripheral iris, one group by a large lens vault (LV), and the third had a mixture of components, respectively. ${ }^{9}$ With the notion that mechanisms contributing to AC might change over time, we hypothesize that the same subgrouping approach will help reveal the factors that become more predominant in the latter stage of the AC disease spectrum, namely, PACG. The purpose of this study was, thus, to identify whether such a subgrouping approach 
was also possible for subjects with PACG, and to determine the characteristics of such subgroups.

\section{Materials and methods}

This prospective observational comparative study was approved by the Institutional Review Board of the hospital and was conducted in adherence to the tenets of the Declaration of Helsinki. Written informed consent was obtained from all the subjects. Subjects aged 40 years and above who had at least one phakic eye diagnosed with PACG were prospectively recruited from glaucoma clinics of the Singapore National Eye Center. Each subject underwent a standardized ophthalmic examination that included an assessment of visual acuity using a logarithm of minimum angle of resolution chart (Lighthouse Inc., Long Island, NY, USA), slitlamp examination, stereoscopic evaluation of the optic disc, and intraocular pressure measurement with Goldmann applanation tonometry (Haag-Streit, Koniz, Switzerland). A-scan ultrasonography (Model US-800; Nidek Co., Ltd., Tokyo, Japan) was used to measure axial length (AL) and lens thickness. Gonioscopy was performed by an experienced examiner in the dark using a Goldmann 2-mirror lens at $\times 16$ magnification. Indentation gonioscopy was carried out using a Sussman 4-mirror lens (Ocular Instruments Inc., Bellevue, WA, USA) to establish the presence and degree of peripheral anterior synechiae (PAS).

PACG was defined as the presence of occludable angles (defined as eyes in which at least $180^{\circ}$ of the posterior pigmented trabecular meshwork was not visible on gonioscopy in the primary position of gaze without indentation) and with glaucomatous optic neuropathy (defined as vertical cup-to-disc ratio $(C D R) \geq 0.7, C D R$ asymmetry $>0.2$, and/or focal notching) with compatible visual field loss on static automated perimetry (SITA Standard algorithm with a 24-2 test pattern; Humphrey Visual Field Analyzer II, Carl Zeiss Meditec, Dublin, California, USA). All subjects had undergone LPI before recruitment into the study. If both eyes of a single patient were eligible, the eye with worse visual field mean deviation (MD) was chosen.

ASOCT (Visante, Carl Zeiss Meditec) imaging was performed for all participants by a single operator, under standardized dark conditions (0 lux). The scans were centered on the pupil and taken along the horizontal axis (nasal-temporal angles at $0-180^{\circ}$ ) using the standard anterior segment single-scan protocol. The examiner adjusted the saturation and noise, and optimized the polarization for each scan during the examination to obtain the best quality image. A single cross-sectional horizontal ASOCT scan of the nasal and temporal angle was evaluated for each subject. The images were processed using customized software, the Zhongshan Angle Assessment Program (ZAAP, Guangzhou, China) by a single experienced observer (MEN) who was masked to clinical data. The only observer input was to determine the position of the scleral spurs. The algorithm then automatically calculated the anterior segment parameters. The parameters measured by ASOCT (Figure 1) were defined as previously. ${ }^{9}$ In brief, anterior chamber width (ACW) was the horizontal scleral spur-to-spur distance

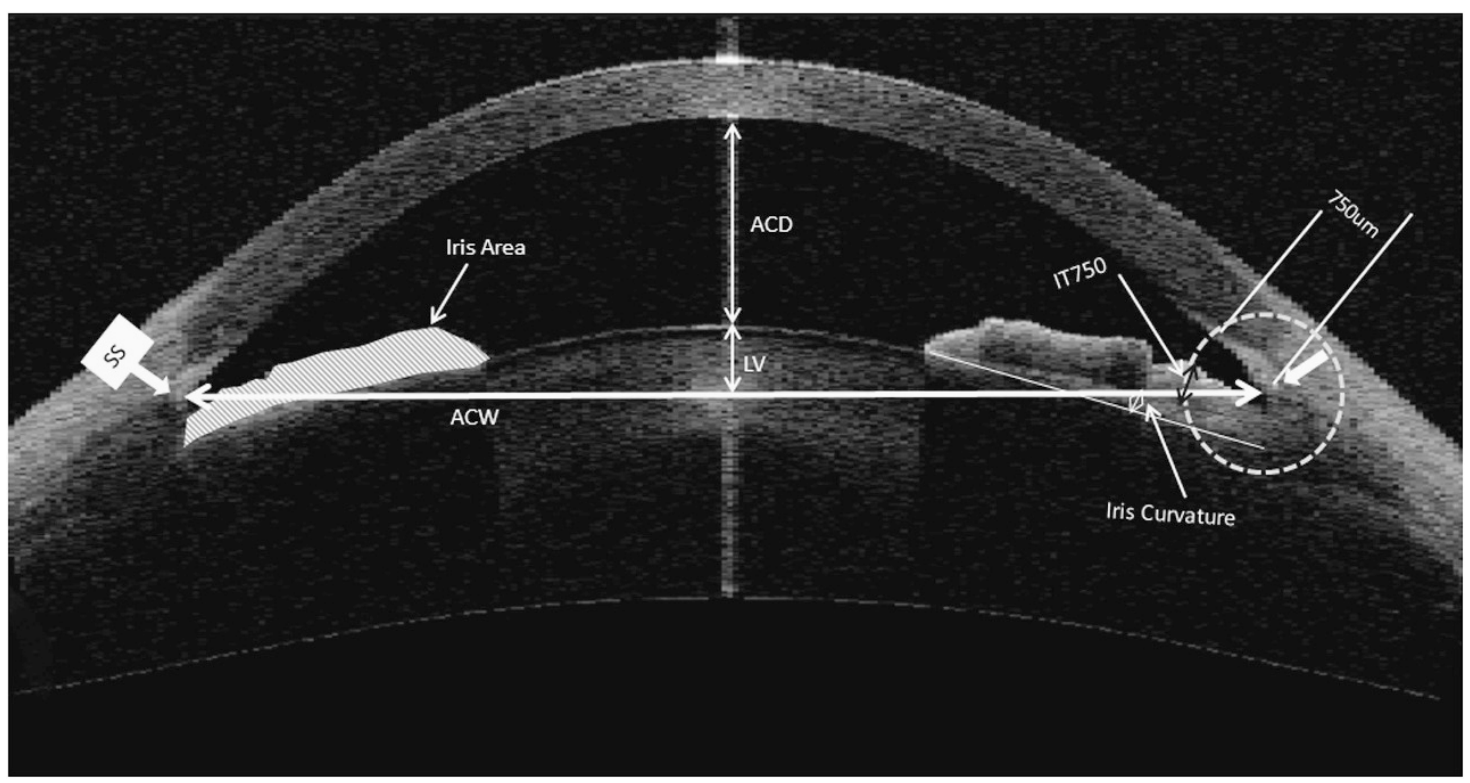

Figure 1 The parameters measured by anterior segment optical coherence tomography (ASOCT) as determined by the Zhongshan Angle Assessment Program. ACD, anterior chamber depth; ACW, anterior chamber width; IArea, iris area; I-Curve, iris curve; IT, iris thickness. 
while LV was the perpendicular distance between the anterior pole of the crystalline lens and the horizontal line joining the two scleral spurs. The iris thickness (IT) 750 and IT 2000 were defined as the IT measured at 750 and $2000 \mu \mathrm{m}$ from the scleral spur, respectively. Iris curvature (ICurv) was the perpendicular distance from a line between the most peripheral to the most central points of the iris pigment epithelium to the posterior surface of the iris at the point of greatest convexity. The iris area (IArea) was calculated as the cumulative cross-sectional area of the full length (from spur to pupil) of the iris. ACA was defined as the cross-sectional area of anterior segment bounded by endothelium, anterior surface of iris, and anterior surface of lens (within the pupil). A vertical axis through the mid-point (centre) of the ACA was plotted by the program, and ACV was calculated by rotating the ACA $360^{\circ}$ around this vertical axis. Pupil diameter (PD) was the shortest distance between the pupil edges of the iris cross-sections.

\section{Statistical analysis}

An agglomerative hierarchical clustering method was first used to determine the optimum number of parameters to be included in the determination of subgroups. Highly correlated parameters were clustered within the same group based on correlations. The agglomerative hierarchical clustering is a bottom-up approach. To begin with, each parameter starts a cluster; then, the pair of clusters with the least inter-cluster distance is merged at each step until only one cluster remain. We used one minus the absolute value of correlation as the distance between the parameters and the Ward method to measure the inter-cluster distance for merging clusters. ${ }^{10}$ After construction of the hierarchical parameter cluster dendrogram, we then determined the optimum number of parameter clusters using the L-method, which finds the number of clusters that optimizes a measure derived from cluster merge. ${ }^{11}$ The parameters were correlated within the same cluster without any correlation across clusters. Therefore, to ensure representativeness of the parameters and yet keep minimum redundancy, we manually chose either one or more parameters from each cluster.

A Gaussian Mixture Model (GMM) ${ }^{12}$ method was then employed to segregate the subjects into subgroups based on the selected parameters. In GMM, all the subjects were assumed to be generated from a superposition of several Gaussian distributions, where each subgroup forms one Gaussian distribution. The most likely subgroup for the PACG cases is then determined by updating the model parameters and case membership in iterations. In this method, the algorithm initializes the centres of the subgroups randomly and calculates the membership probability of each subject to a given subgroup. A subject is then assigned to the subgroup with the highest membership probability. Depending on the new subject allocation, the algorithm then updates the subgroup centres accordingly. The process of calculating the subject-to-subgroup membership probability, allocating subjects to respective subgroups, and updating subgroup centres were repeated until the allotment of the subjects no longer changed. The GMM was executed under different random initializations, and they converged to the same clustering result in over $90 \%$ of the different initializations. The converged clustering was chosen as the final result for this setting. The GMM was also executed multiple times to put the cases into two, three, four, or five subgroups, respectively, and the optimal number of subgroups was then determined using the corrected Akaike Information Criterion (AIC), which is a measure of relative goodness-of-fit of a statistical model. ${ }^{13}$ Under AIC, a simple model with high likelihood is favored.

In addition, each parameter in the subgroup was qualitatively categorized into large, medium, or small, depending on the differences of their absolute value. Quantitative variables were compared using one-way analysis of variance, and Bonferroni adjustment was applied for pair-wise comparisons.

\section{Results}

Of 237 subjects identified to have PACG, 27 were excluded due to poor quality images, or software delineation error, or indeterminate sclera spur, leaving 210 subjects with complete data for the final analysis. The mean age (s.d.) of the 210 subjects was 67.9 (9.2) years and $53.3 \%$ were female. Subgroup 1 had a larger mean AL $(23.46 \pm 1.05 \mathrm{~mm})$ when compared with subgroup 2 $(22.75 \pm 0.83 \mathrm{~mm}, P<0.001)$ and subgroup 3 $(22.82 \pm 0.94 \mathrm{~mm}, P=0.006)$. The difference between subgroup 2 and 3 was not statistically significant $(P=0.99)$. Mean visual field MD and CDR of the overall sample were $-12.5 \pm 9.0$ and $0.8 \pm 0.1 \mathrm{~dB}$, respectively. PAS was observed in 107 eyes (51\%), and the mean total PAS in these patients was $3.6 \pm 2.5$ clock-hours. Figure 2 shows the hierarchical parameter clustering (dendrogram) and L-method analysis. As the iris parameters are clustered together and correlated with PD, we chose only one parameter, IArea, from this parameter cluster. From the second cluster, we chose LV, anterior chamber depth (ACD), and ACW, as they are farther apart in the dendrogram cluster.

Based on the GMM analyses, we found that the optimal number of subgroups of PACG, as obtained by the lowest AIC was 3. With four or five subgroups, the model was complex and less favored by AIC, and with only two subgroups, the model was simple but the grouping had 


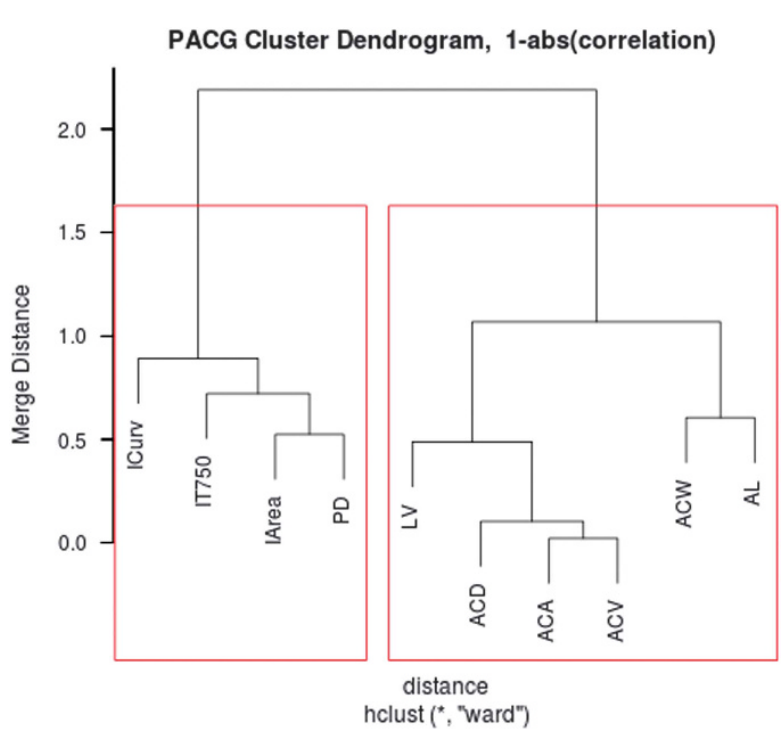

Figure 2 Hierarchical parameter clustering/dendrogram for the PACG subjects. Correlated features are grouped in the same cluster bounded by the red box in the dendrogram. ACA, anterior chamber area; ACD, anterior chamber depth; ACV, anterior chamber volume; $\mathrm{ACW}$, anterior chamber width; $\mathrm{AL}$, axial length; IArea, iris area; ICurv, iris curvature; IT, iris thickness; LV, lens vault; PD, pupil diameter.

very low likelihood. Table 1 shows the subgrouping result of the PACG cases. Subgroup 1 was characterized by a larger IArea, subgroup 2 by a large $\mathrm{LV}$, and a shallow ACD, whereas subgroup 3 displayed only intermediate values across IArea, $\mathrm{LV}$, and ACD. The proportion of eyes classified into subgroup 2 was the least $(N=24 ; 11.4 \%)$, followed by subgroup $1(N=89 ; 42.4 \%)$ and subgroup 3 $(N=97 ; 46.2 \%)$, respectively (Table 1$)$. There were no significant differences in the mean age $(P>0.05)$, visual $\mathrm{MD},(P>0.05)$ pattern standard deviation (PSD, $P>0.05)$, and vertical CDR $(P>0.05)$ between the three subgroups.

\section{Discussion}

Mirroring our findings for PACS, we also identified three distinct subgroups of PACG subjects based on ASOCT imaging. Although the characteristics of the subgroups and the trend observed in PACG subjects were similar to our previous findings, the proportional distribution of patients across the subgroups was different. ${ }^{9}$ The proportion of PACG subjects assigned to the subgroup that comprise of intermediate elements (subgroup 3) was the largest (46.2\%), followed by the subgroup characterized by larger IArea $(42.4 \%)$ and a relatively small LV with deep anterior chambers (subgroup 1). The lowest proportion was observed in the subgroup that demonstrated a large LV and small ACD (subgroup 2, 11.4\%).

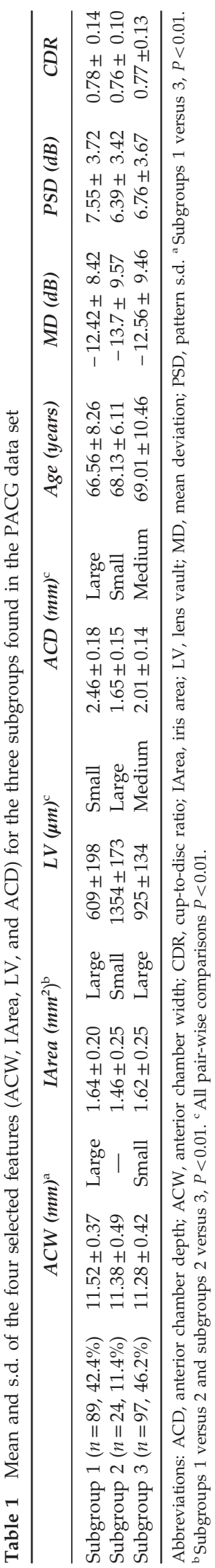


Notably, a higher proportion of PACG (42.4\%) patients were categorized in subgroup 1 compared with PACS $(30 \%),{ }^{9}$ the earliest stage in AC disease spectrum. The most noticeable component in this subgroup is the large IArea with rather less contributions from LV and ACD. These eyes also had significantly larger ALs when compared with the other two groups. It is likely that IArea may have been overestimated due to PAS, which might have caused the iris cross-sectional area look larger by pulling the iris towards the angle. However, considering that only half of the patients exhibited PAS, we presume this effect to cause minimal, if any, change in IArea. Furthermore, considering that this subgroup displayed relatively larger ACD and smaller LV, plateau iris may be the mechanism in these patients. Laser iridoplasty may be beneficial in patients with large IArea and residual AC, as this procedure thins and flattens the peripheral iris reducing the surface area that comes into appositional contact with the angle upon physiologic pupil dilation. ${ }^{14}$ IArea, as a surrogate for iris volume, along with ICurv has been found to be independently associated with the presence of narrow angles in untreated patients (with no previous history of LPI) in a cross-sectional community-based study. ${ }^{15}$ As it is well documented that ICurv decreases significantly after LPI, whereas IArea does not, ${ }^{16}$ it is tempting to speculate that larger IArea may be a risk factor for progressive closure of the angle once the pupillary block is relieved after LPI. ${ }^{16,17}$ The mechanism by which larger IArea contributes to AC may possibly be ascribed to the dynamic properties of the iris. Several studies have shown that the iris loses less fluid and retain its volume after pupillary dilation in eyes with AC. ${ }^{18-20}$ In a mixed population, including those subjects with previous LPI, Seager et $\mathrm{al}^{21}$ found that larger baseline IArea and less iris volume loss with mydriasis increased the likelihood of having AC, particularly in Chinese persons. Thus, it may be hypothesized that mechanisms leading to appositional obstruction of the trabecular meshwork in subgroup 1 may be related to a possible interaction between a larger iris volume and disturbances in dynamic iris behavior.

The main determining components in subgroup 2 are ACD and LV. Shallower ACD and greater LV have been found to be independently associated with AC. 6,22 Although LV explains $58 \%$ of the variability in ACD and they are logically thought to be inversely correlated to each other, we chose to include both of them, as they were further away from each other in the clustering dendrogram. ${ }^{23}$ The LV has a distinct role in AC pathogenesis: a more anteriorly positioned and thickened lens may contribute to anterior chamber shallowing and may push the peripheral iris against the trabecular meshwork, thereby crowding the angle, or the increased bulk of the lens may favor more iris-lens contact further aggravating the pupil block. ${ }^{24}$ Interestingly, Han et al ${ }^{25}$ found that AC eyes with shallower ACD and a more prominent LV, displayed greater angle widening postLPI compared with the group with relatively deeper anterior chamber and smaller LV. This differential effect of LPI was attributed to plateau iris configuration (as speculated by a deeper ACD) in the latter group. ${ }^{25}$ They suggested that LPI was more successful in eliminating the pupil block component in the former group. ${ }^{25}$ In sum, it is conceivable that AC is mainly driven by a large LV and a small ACD in subgroup 2, whereas in subgroup 3, there are no predominant anatomical features. It is possible that a mixed mechanism involving elements of both subgroup 1 and 2 might be responsible in the third subgroup.

In our analysis, we followed a methodical and unbiased approach wherein the most appropriate parameters were first identified based on correlations and hierarchical clustering. This was followed by the ascertainment of the optimum number of subgroups using GMM and AIC. Within and between the subgroups, each parameter had varying amounts of overall influence. This suggests that these subgroups likely represent different mechanisms involved in the development of AC. Of note, glaucoma severity (MD, PSD, and CDR) was similar across the three subgroups, implying that segregation was purely based on inherent anatomical characteristics. We believe that this approach may be used to determine the predominant mechanism underlying $\mathrm{AC}$ in a particular patient and thereby serving as a guide to individualize treatment. More studies are needed to provide convincing data that would necessitate modification of the existing AC management strategy/guidelines and an added ability to subcategorize eyes based on the predominant anatomical mechanism may help guide in clinical decision-making.

The strengths of this study include the relatively large sample size of PACG subjects, and application of a standardized and objective statistical method for subgrouping. The study is limited by being based on a sample population consisting mostly of Chinese persons. In addition, our analyses only relied on static measurements from one meridian as obtained from the ASOCT, which is incapable of visualizing structures posterior to the iris. This has precluded assessment of the influence of dynamic factors as well as the impact of ciliary body in the subgrouping.

In conclusion, this study has shown that with concurrent assessment of the anterior segment parameters, it is possible to determine the predominant anatomical component(s) in PACG. These results may provide a framework for improving treatment strategies based on subdivisions of PACG. 


\section{Summary}

\section{What was known before}

- It has been previously shown that primary angle-closure suspects (PACSs) can be subgrouped into three distinct subgroups based on anterior segment optical coherence tomography (ASOCT) imaging and clustering analysis. However, it is not known if is possible to subgroup eyes with primary angle-closure glaucoma (PACG) and what are the characteristics of such subgroups (if any).

\section{What this study adds}

- This study shows that PACG eyes can also be divided into three distinct subgroups. However, the proportional distribution of PACG eyes across the subgroups was different from PACS.

\section{Conflict of interest}

The authors declare no conflict of interest.

\section{Acknowledgements}

Supported by the Singapore Ministry of Health's National Medical Research Council, under its Singapore Translational Research Investigator Award (NMRC/STAR/0023/2014).

\section{References}

1 Chan EW, Li X, Tham YC, Liao J, Wong TY, Aung T et al. Glaucoma in Asia: regional prevalence variations and future projections. Br J Ophthalmol 2016; 100(1): 78-85.

2 Foster PJ, Machin D, Ng T-P, Devereux JG, Johnson GJ, Khaw PT et al. The prevalence of glaucoma in chinese residents of singapore a cross-sectional population survey of the tanjong pagar district. Arch Ophthalmol 2000; 118: 1105-1.

3 Quek DT, Koh VT, Tan GS, Perera SA, Wong TT, Aung T. Blindness and long-term progression of visual field defects in chinese patients with primary angle-closure glaucoma. Am J Ophthalmol 2011; 152(3): 463-469.

4 He M, Friedman DS, Ge J, Huang W, Jin C, Lee PS et al. Laser peripheral iridotomy in primary angle-closure suspects: biometric and gonioscopic outcomes: the Liwan Eye Study. Ophthalmology 2007; 114(3): 494-500.

5 Jiang Y, Chang DS, Zhu H, Khawaja AP, Aung T, Huang S et al. Longitudinal changes of angle configuration in primary angle-closure suspects: the Zhongshan Angle-Closure Prevention Trial. Ophthalmology 2014; 121(9): 1699-1705.

6 Nongpiur ME, He M, Amerasinghe N, Friedman DS, Tay WT, Baskaran $\mathrm{M}$ et al. Lens vault, thickness, and position in Chinese subjects with angle closure. Ophthalmology 2011; 118(3): 474-479.

7 Foo LL, Nongpiur ME, Allen JC, Perera SA, Friedman DS, $\mathrm{He} \mathrm{M}$ et al. Determinants of angle width in Chinese Singaporeans. Ophthalmology 2012; 119(2): 278-282.

8 Wu RY, Nongpiur ME, He MG, Sakata LM, Friedman DS, $\mathrm{Chan} \mathrm{YH}$ et al. Association of narrow angles with anterior chamber area and volume measured with anterior-segment optical coherence tomography. Arch Ophthalmol 2011; 129(5): 569-574.
9 Nongpiur ME, Gong T, Lee HK, Perera SA, Cheng L, Foo LL et al. Subgrouping of primary angle-closure suspects based on anterior segment optical coherence tomography parameters. Ophthalmology 2013; 120(12): 2525-2531.

10 Ward JH. Hierarchical grouping to optimize an objective function. J Am Stat Assoc 1963; 58: 236-244.

11 Salvador S, Chan P Determining the number of clusters/ segments in hierarchical clustering/segmentation algorithms. 16th IEEE International Conference on Tools with Artificial Intelligence (ICTAI 04); Institute of Electrical and Electronics Engineers, Inc.: Piscataway, NJ, USA, 2004, pp 576-584.

12 GJ McLachlan, Peel D. Multivariate normal mixtures. In: Shewhart WA, Wilks SS (eds). Finite Mixture Models. Wiley: New York, Ny, USA, 2000, pp 81-116.

13 Hurvich CM, Tsai CL. Regression and time series model selection in small samples. Biometrika 1989; 76: 297-307.

14 Ritch R, Tham CC, Lam DS. Argon laser peripheral iridoplasty (ALPI): an update. Surv Ophthalmol 2007; 52(3): 279-288.

15 Wang B, Sakata LM, Friedman DS, Chan YH, He M, Lavanya R et al. Quantitative iris parameters and association with narrow angles. Ophthalmology 2010; 117(1): 11-17.

16 How AC, Baskaran M, Kumar RS, He M, Foster PJ, Lavanya R et al. Changes in anterior segment morphology after laser peripheral iridotomy: an anterior segment optical coherence tomography study. Ophthalmology 2012; 119(7): 1383-1387.

17 Lee RY, Kasuga T, Cui QN, Porco TC, Huang G, He M et al. Association between baseline iris thickness and prophylactic laser peripheral iridotomy outcomes in primary angleclosure suspects. Ophthalmology 2014; 121(6): 1194-1202.

18 Quigley HA, Silver DM, Friedman DS, He M, Plyler RJ, Eberhart CG et al. Iris cross-sectional area decreases with pupil dilation and its dynamic behavior is a risk factor in angle closure. J Glaucoma 2009; 18(3): 173-179.

19 Aptel F, Denis P. Optical coherence tomography quantitative analysis of iris volume changes after pharmacologic mydriasis. Ophthalmology 2010; 117(1): 3-10.

20 Zhang Y, Li SZ, Li L, He MG, Thomas R, Wang NL. Quantitative analysis of iris changes following mydriasis in subjects with different mechanisms of angle closure. Invest Ophthalmol Visual Sci 2015; 56(1): 563-570.

21 Seager FE, Jefferys JL, Quigley HA. Comparison of dynamic changes in anterior ocular structures examined with anterior segment optical coherence tomography in a cohort of various origins. Invest Ophthalmol Visual Sci 2014; 55(3): $1672-1683$.

22 Ozaki M, Nongpiur ME, Aung T, He M, Mizoguchi T. Increased lens vault as a risk factor for angle closure: confirmation in a Japanese population. Graefes Arch Clin Exp Ophthalmol 2012; 250(12): 1863-1868.

23 Sng CC, Foo LL, Cheng CY, Allen Jr JC, He M, Krishnaswamy G et al. Determinants of anterior chamber depth: the Singapore Chinese Eye Study. Ophthalmology 2012; 119(6): 1143-1150.

24 Tarongoy P, Ho CL, Walton DS. Angle-closure glaucoma: the role of the lens in the pathogenesis, prevention, and treatment. Surv Ophthalmol 2009; 54(2): 211-225.

25 Han S, Sung KR, Lee KS, Hong JW. Outcomes of laser peripheral iridotomy in angle closure subgroups according to anterior segment optical coherence tomography parameters. Invest Ophthalmol Visual Sci 2014; 55(10): 6795-6801. 